\title{
Assisting control of forces in laparoscopy using tactile and visual sensory substitution
}

\author{
Thomas Howard ${ }^{1}$ and Jérôme Szewczyk ${ }^{2}$ \\ Sorbonne Universités, UPMC Univ Paris 06, ISIR, CNRS, UMR 7222, INSERM, \\ U1150, Agathe-ISIR, F-75005, Paris, France \\ e-mail: howard@isir.upmc.fr
}

\begin{abstract}
It is widely recognized that despite its many benefits, the ergonomics of laparoscopic surgery lead to severe perceptual distortions for the surgeon. In particular, interaction forces at the instrument tip are masked by interfering forces at the trocar and distorted due to a lever effect around the insertion point. This leads to improper control of tool-tip interaction forces, increasing the risk and occurrence of intra-operative injuries, unnecessary trauma to healthy tissue and suture breakage.

Here, we propose an experiment aimed at determining the efficacy and usability of cutaneous vibrotactile and/or visual feedback of tool-tip interaction forces in assisting a surgeon in controlling fine interaction forces. 16 novice subjects performed force-reach and -hold tasks in a laparoscopic setting under provision of 9 forms of feedback (visual feedback, 4 variants of vibrotactile feedback, and their combinations).

Feedback increased precision (up to $85.8 \%$ reduction in error when aiming for a target force), repeatability (up to $84 \%$ reduction in spread of aiming errors), speed of reaching a target force (up to 18 -fold increase in speed of reaching a target force at equal accuracy) and reduced force drift over time ( $>68 \%$ reduction in cumulative deviation from a target force over a 20 s period). Results show best performance for visual feedback, with promising performance for pulsed vibrotactile feedback, allowing us to draw initial conclusions on the potential for using tactile feedback to represent interaction forces in laparoscopy and to gain insights into axes for its improvement.
\end{abstract}

Key words: haptics, laparoscopy, force feedback, sensory substitution, MIS

\section{Introduction}

Laparoscopic surgery (LS) is widely used to treat a variety of pathological conditions ([4], [9], [14]) boasting many advantages for the patient due to the reduced access trauma. However, the deteriorations in dexterity and perception stemming from the manipulation of thin elongated instruments inserted into the abdomen through trocars coupled with vision from a 2-D endoscopic camera are the cause of suboptimal performance on the part of surgeons [19]. This sometimes results in unnecessary complications ([15], [3], [5], [2]). Among the most cited problems in LS, distorted and partially lost haptic perception of forces is a major cause for concern 
([13], [16], [10]), prompting us to seek solutions for assisting surgeons in better controlling them.

Haptic perception of forces is also a major issue in robotically assisted minimally invasive surgery (RMIS), as teleoperation has only worsened the situation compared to LS, exacerbating the problem of excessive applied forces. An interesting overview of force feedback in RMIS is given by Weber et al. [17], who perform a meta-analysis of 21 studies comparing surgical task performance with and without force feedback. They conclude that force feedback has a moderate effect on accuracy, a strong beneficial effect on the control of average and peak forces and no effect on task completion times. However, technical difficulties in implementing force feedback for RMIS have prompted research in alternate methods for presenting force information, in particular through sensory substitution.

Both audio ([7], [8], [1]) and visual feedback in RMIS ([7], [11]) and LS (e.g. [18]) have proven effective in reducing mean and peak forces during suturing and tissue manipulation. However, implementing audio feedback in the operating room where ambient noise is high and it may impede communication remains challenging. Visual feedback requires a possibly distracting display alongside the endoscopic image, and increasing the already high visual cognitive load.

For this reason, tactile feedback has been considered as an option for displaying interaction forces, with the advantage of not competing with other important information passing through the same perceptual channel, and providing immediate, private and non-disruptive cues. To the best of our knowledge, most work on tactile feedback for LS has focussed on cutaneous feedback of grasping forces ([18], [12]) and tissue palpation forces ([20]) with promising results.

In the case of suture knot tying, there is great benefit to be obtained from better control of interaction forces at the tool-tip. Sutures are safe as long as they exceed a minimum thread tension when tied, ensuring good closure of the wound. On the other hand, excess suture thread tension leads to tissue damage or suture breakages and associated complications ([18]).

Here, we propose an experiment aimed at determining whether it is possible to represent tool-tip interaction forces through haptic and/or visual cues in a manner that is intuitively usable to better control them, seeking insight into the best form for representing forces. Subjects are asked to apply and hold pre-defined levels of suture thread tension under provision of visual and/or vibrotactile feedback.

Section 2 describes our experimental set-up and protocol. Results obtained are shown in section 3 and subsequently discussed for each evaluation metric. Finally, general conclusions are drawn in section 4 and axes for future work are outlined.

\section{Materials and methods}

Our experiment focussed on the manipulation of a suture thread attached to the end of a laparoscopic instrument, with the aim of subjecting it to given levels of tension forces. 16 Subjects (10 male, 6 female, all novices in laparoscopy) performed a se- 
quence of force reach and hold tasks for a selection of 10 feedback conditions.

\subsection{Experimental Hardware}

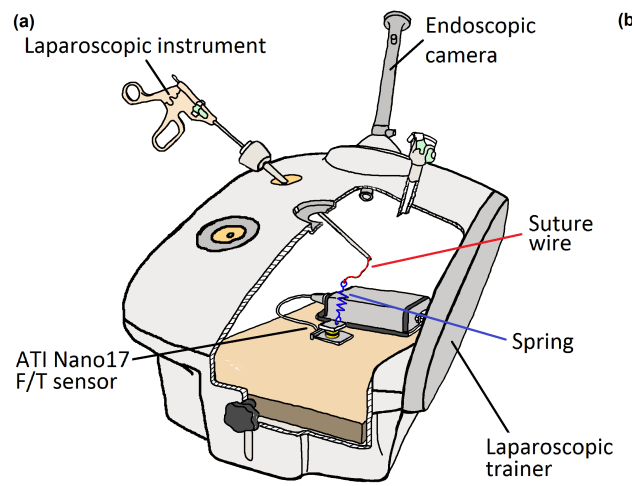

(b)

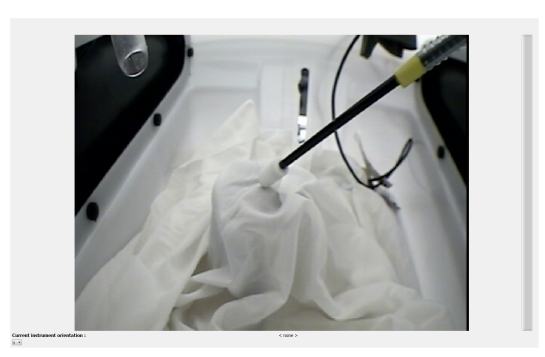

Fig. 1 (a) Experimental setup; (b) Endoscopic image as seen by the subject

Subjects were placed in front of a laparoscopic trainer (EndoSim LaproTrain) containing a plate equipped with a force sensor with a fixture for holding a suture wire (3-0 gauge Braun Novosyn 90/10), as shown in fig. 1 left. They manipulated standard laparoscopic forceps with the suture wire attached so as to avoid time losses associated with novices attempting to grasp a suture thread. Fig. 1 left highlights the force sensor (ATI Nano17E 6-axis force/torque sensor, sensing range [0N-12N], force values acquired at $40 \mathrm{~Hz}$ ) in yellow, the spring between the force/torque sensor and suture thread in blue and the suture thread in red. The role of the spring was to introduce a relatively low stiffness elastic component between the highly stiff wire and force sensor, mimicking the natural elasticity of tissue during suture knot tying or tissue manipulation. Subjects observed the scene on a 24" LCD monitor placed directly in front of them, and manipulated the laparoscopic forceps through a 5mm trocar, observing the inside of the laparoscopic trainer as shown in Fig. 1 right. Loose white sheets were placed over the force/torque sensor and spring so as to minimize visual cues that could aid in assessing the thread tension. Vibrotactile feedback was provided using an ERM motor (Precision Microdrives PicoVibe 307100 , vibration intensity range $[0 \mathrm{~g}-7 \mathrm{~g}]$ strapped to the inside of the subject's hand so as to simulate integration of feedback to a LS tool handle. New feedback commands were generated at a frequency of $40 \mathrm{~Hz}$, following the acquisition of the force data. 


\subsection{Feedback conditions}

Reference - No feedback (L) : Subjects received no feedback about the suture thread tension $F_{\text {tension }}$ and relied solely on the visual information from the endoscope and natural tactile information from the instrument.

Visual feedback $(\mathbf{V})$ : Subjects received visual feedback about $F_{\text {tension }}$ in the form of a vertical bar-graph displayed next to the endoscopic image. The bar-graph displayed forces in the range [0N-3N]. We did not highlight the target force in any way. The feedback level as a function of $F_{\text {tension }}$ is shown in green in fig. 2 (a).

Continuous vibrotactile $(\mathbf{T})$ : Subjects received tactile feedback about $F_{\text {tension }}$ through an ERM vibration motor. The tension force $F_{\text {tension }}$ was displayed through a linearly proportional variation of vibration amplitude ( $0 \mathrm{~g}$ to $5.2 \mathrm{~g}$ ), as shown in red in fig. 2 (a).

Pulsed vibrotactile - fixed pulse length (Uf): The same vibration motor as for condition $\mathrm{T}$ displayed the suture tension $F_{t}$ though series of vibration pulses at a fixed amplitude $(3.4 \mathrm{~g})$ and length $(45 \mathrm{~ms})$ with a spacing inversely proportional to the applied force varying between $525 \mathrm{~ms}$ and $15 \mathrm{~ms}$, as shown in red in fig. 2 (b).

Pulsed vibrotactile - varying pulse length and interval (Up): Identical set-up to Uf, however this time the length and spacing of the pulses were varied jointly in the same manner as the spacing in Uf, always keeping spacing and length equal. The pattern of force encoding is shown in red in fig. 2 (d).

Pulsed vibrotactile - fixed pulse interval (Uq): Identical set-up to Uf, however this time the pulse spacing was kept constant at $45 \mathrm{~ms}$, and the pulse length was varied between $525 \mathrm{~ms}$ and $15 \mathrm{~ms}$ in inverse relation to the applied suture tension, as shown in red in fig. 2 (c).

Vibrotactile and visual conditions (TV, UfV, UpV and UqV): These four conditions are identical to T, Uf, Up and Uq respectively, whereby we simultaneously provide visual feedback as in condition $\mathrm{V}$.

\subsection{Task}

In chronological order, the task to be performed consisted of:

- 1 definition of a target force between 1 and $3 \mathrm{~N}$ : Subjects pulled on the suture wire while receiving feedback corresponding to the given condition and pressed a button to define the current force as their target for the rest of the task (referred 

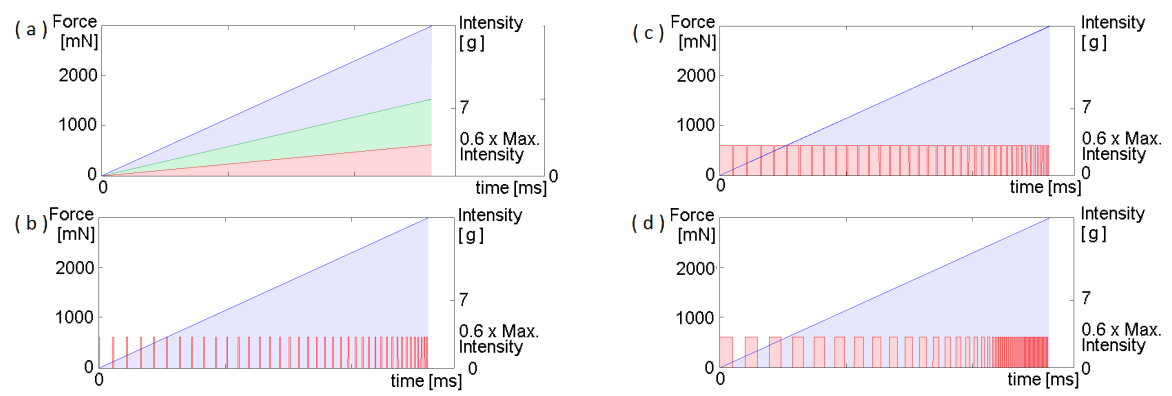

Fig. 2 Feedback levels depending on the magnitude of force to be displayed. From the top left to bottom right : (a) Visual (V) and continuous vibrotactile feedback (T); (b) Pulsed vibrotactile feedback (Uf); (c) Pulsed vibrotactile feedback (Uq); (d) Pulsed vibrotactile feedback (Up). The blue curve indicates the force magnitude, the green curve the associated bargraph heigh and the red curve the vibration intensity.
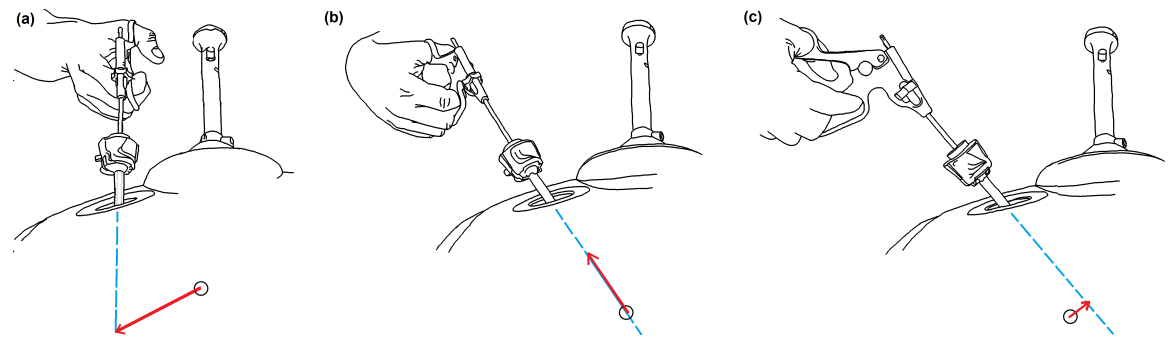

Fig. 3 Extreme cases of tool orientations when pulling on the suture wire. The blue dotted line shows the instrument axis, the red arrow the suture thread axis. From left to right : pulling on the wire using lever effect with instrument parallel to the saggital plane (a), directly pulling on the wire with intrument and wire aligned on the trocar axis (b), and pulling on the wire using lever effect with instrument parallel to the coronal plane

to as the actual target force $F_{t g t}$ in the following). Subjects were under no time pressure to select a target force.

- 1 force reach task, where the objective was to aim for the previously defined $F_{t g t}$, having changed the instrument's orientation (see fig. 3 ) in order to minimize the impact of visual cues in the endoscopic image on assessment of $F_{t}$. The subjects pressed a button once they thought they had reached $F_{t g t}$ (in the following, the force applied at the moment of this button press will be referred to as the estimated target force $F_{\text {tgt, est }}$ ).

- 1 force hold task, where the objective was to hold $F_{\text {tgt,est }}$ for a duration of 20s.

- 3 force repeat tasks, where the objective was once again to aim for $F_{t g t}$ as precisely as possible, changing the instrument orientation every time. For this, the subject pressed a button to begin, pulled on the thread, and pressed the button again once $F_{t g t}$ was thought to have been reached.

To keep the experiment duration manageable, subjects were assigned a randomized sequence of the conditions L, V, T, Uf, Up, Uq, and one of the four vi- 
sual+tactile conditions (TV, UfV, UpV or UqV). For each condition, subjects performed two to three repeats of the task previously described. They were each given detailed instructions about the experimental sequence and manipulation of the laparoscopic instrument. Furthermore, they were briefed and given an example for each form of feedback prior to performing the task.

\subsection{Evaluation metrics}

Mean distance to target (DTT) : We calculated DTT as the mean of differences between $F_{t g t}$ and $F_{t g t, e s t}$ for each force reach and force repeat task for each subject.

$$
D T T_{\text {Subject }}=\frac{\sum_{i=1}^{N}\left(F_{\text {tgt }}(i)-F_{\text {tgt }, e s t}(i)\right)}{N}[\mathrm{mN}]
$$

where $N=$ no. of force reaches + no. of force repeats performed by the subject.

Values close to 0 indicate higher aiming accuracy. Lower spread indicates greater repeatability. Dividing subject DTT for each condition by the subject's DTT for condition L highlights improvements (values $<1$ ) or deteriorations (values $>1$ ) from the provided feedback while compensating for differences in subject's natural capabilities in laparoscopic instrument manipulation.

\section{Speed of aiming (SFR) :}

We evaluated the speed of aiming at a target force for a given final accuracy.

$$
S F R=\frac{1}{N} \sum_{i=1}^{N} \frac{F_{t g t}(i)}{(T C T(i) *|D T T(i)|)}\left[s^{-1}\right]
$$

Larger values indicate lower DTT at equal reach speeds or higher reach speeds at equal DTT. We evaluate mean subject SFR for each condition divided by subject SFR in condition L to compensate for subject's natural capabilities in manipulating the laparoscopic instrument.

\section{Mean error during force-hold task (FHE) :}

FHE is the mean of differences between the subject's mean force applied during the force-hold task and $F_{t g t, e s t}$ from the initial force-reach task for a given condition:

$$
F H E=\frac{1}{K} \sum_{i=1}^{K} F_{\text {tgt,est }}(i)-\text { mean }\left(F_{\text {tension }}(i)\right)[m N]
$$

where $\mathrm{K}$ is the number of force-hold tasks performed by the subject.

\section{Cumulative deviation (CD) :}

$\mathrm{CD}$ is calculated as the mean of the integral of applied force over the duration of force hold tasks for a given feedback condition: 


$$
C D=\frac{1}{K} \sum_{i=1}^{K}\left(\sum_{j=1}^{\text {durationofforcehold }} F_{\text {tension }}(i, j)\right)[N . s]
$$

This metric is representative of amplitude of oscillations around the target force and drift. Larger values indicate worse performance.

\section{Drift angles :}

Mean drift for a given condition is calculated by fitting a line to the curve of applied force during the force-hold task and evaluating its slope (the drift angle - D $\alpha$ - in $[\mathrm{N} / \mathrm{s}])$. Values further away from zero indicated a marked tendency to drift away from $F_{t g t, e s t}$. The sign of the drift angles also gives an indication of the direction of drift, with positive values indicating a tendency to apply more force over time and negative values indicating a tendency to reduce applied forces over time.

\section{Results and discussion}

In the following, we present and discuss the main results obtained for the various evaluation metrics described earlier.

\subsection{Precision and repeatability of reaching a target force}

Fig. 4 (a) shows that providing feedback systematically reduces mean subject DTT (see table 1 for subject mean DTT), all differences in means significant with at least $\mathrm{p}<0.05)$.
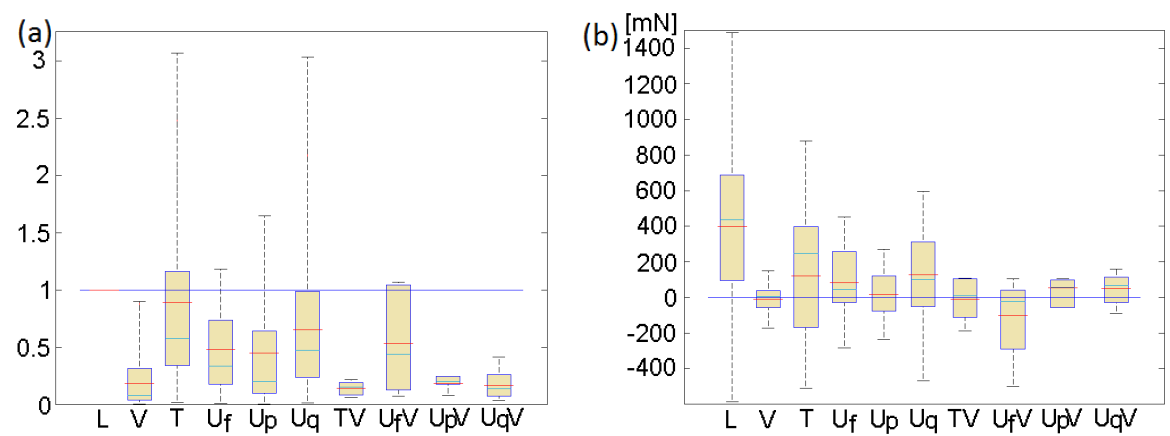

Fig. 4 (a) : Mean distance to target for force reach and repeat tasks for each subject, divided by the subject's mean DTT in condition (L) [dimensionless]. Medians are indicated by the light blue and means by the red horizontal lines; (b) Mean DTT for each subject, grouped by condition [mN].

The best performance is obtained for conditions $\mathrm{V}(81.77 \%$ reduction in mean error over condition $\mathrm{L}, \mathrm{p}<0.01)$, TV $(85.8 \%$ reduction in mean error over condition 
$\mathrm{L}, \mathrm{p}<0.01), \mathrm{UpV}(81.98 \%$ reduction in mean error over condition $\mathrm{L}, \mathrm{p}<0.01)$ and $\mathrm{UqV}(83.23 \%$ reduction in mean error over condition $\mathrm{L}, \mathrm{p}<0.01)$, with a systematic improvement of performance over condition $\mathrm{L}$ for all subjects. We believe the better performance obtained through visual feedback can be attributed to the fact that it not only provides information on the current force magnitude but also provides relative distances to the maximum and minimum levels of feedback at any given time, whereas the level of tactile feedback is harder to place on the range of possible feedback levels.

Vibrotactile feedback alone leads to moderate improvements, with the best performance achieved for Uf (52.21\% reduction in mean error over L, improvements in $>75 \%$ of cases) and Up $55.07 \%$ reduction in mean error over $\mathrm{L}$, improvements in $>75 \%$ of cases), although in some rare cases, subjects did not succeed in performing better with them than with visual feedback alone. It seems that vibrotactile pulses yields higher sensitivity to changes than continuous vibration. Also, this difference could relate to greater user comfort when vibration was presented in short bursts. Indeed, condition T shows the worst performance of all tactile conditions, with improvements over $\mathrm{L}$ observed $<75 \%$ of the time.

In order to further analyse the effect of feedback on errors when aiming for predefined target forces, fig. 4 (b) shows the mean DTT values for each subject. In the absence of feedback, there is a marked tendency to not reach the predefined target force. This graph allows analysis of the effect of feedback on repeatability of aiming, shown by the spread of values for each condition (see table 1 for CD means and standard deviations (SD)). Improvements in mean errors generally hold true for spread, with the best repeatability obtained for conditions $\mathrm{V}(62.8 \%$ reduction in SD over condition L), Up (52.4\% reduction in SD over condition L), TV (77.7\% reduction in SD over condition $\mathrm{L})$ and $\mathrm{UqV}(84.7 \%$ reduction in SD over condition $\mathrm{L})$. Conditions Uf and UpV yield moderate improvements in spread (42.2\% and $44.7 \%$ reduction in SD over condition L respectively) while the remaining conditions yield only very slight improvements.

In terms of precision, there seems to be no great use in combining visual and tactile feedback (except for the improvement between UqV and Uq, $\mathrm{p}<0.05$ ), as the combination is either detrimental to performance or the tactile feedback is clearly ignored in favour of the visual feedback. The only positive impact that can be noted is a slight improvement in terms of repeatability, which can probably be attributed to the redundancy of information.

Table 1 Mean subject DTTs for each condition [mN]

\begin{tabular}{lllllllllll}
\hline & $\mathrm{L}$ & $\mathrm{V}$ & $\mathrm{T}$ & $\mathrm{Uf}$ & $\mathrm{Up}$ & $\mathrm{Uq}$ & $\mathrm{TV}$ & $\mathrm{UfV}$ & $\mathrm{UpV}$ & $\mathrm{UqV}$ \\
\hline Means : & 398.91 & -8.996 & 121.64 & 82.94 & 15.58 & 127.57 & -11.3 & -102.1 & 52.16 & 51.02 \\
SDs : & 531.79 & 197.6 & 375.21 & 307.54 & 253.2 & 309.87 & 118.45 & 204.9 & 293.96 & 81.85 \\
\hline
\end{tabular}




\subsection{Speed of reaching a target force}

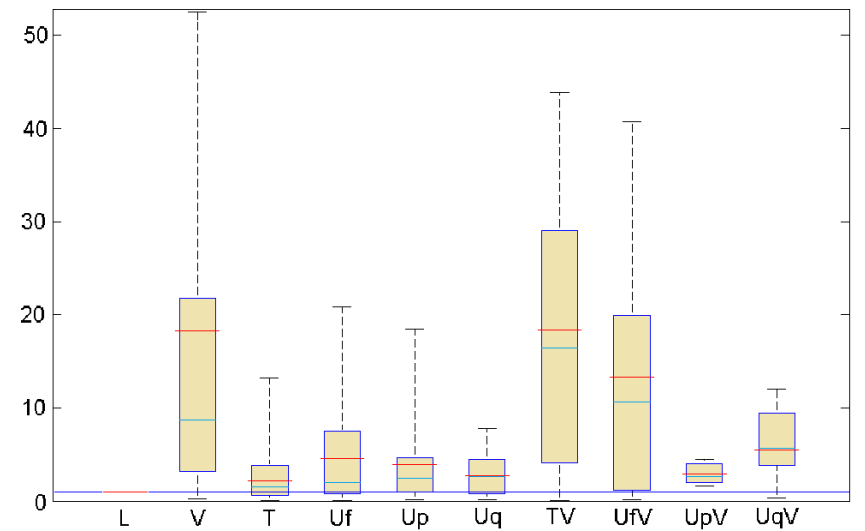

Fig. 5 Mean subject speed of reaching a target force (SFR) for a given DTT, relative to subject's performance in condition L [dimensionless].

Feedback significantly increases SFR at identical DTT $(\mathrm{p}<0.05)$ except for conditions $\mathrm{T}, \mathrm{UpV}$ and $\mathrm{UqV}$. Table 2 sums up the mean values for each condition.

Table 2 Subject means for speed of reaching a target force for a given DTT, relative to subject's performance in condition L [dimensionless], against subject mean TCT in each condition [s]

\begin{tabular}{lllllllllll}
\hline & $\mathrm{L}$ & $\mathrm{V}$ & $\mathrm{T}$ & $\mathrm{Uf}$ & $\mathrm{Up}$ & $\mathrm{Uq}$ & $\mathrm{TV}$ & $\mathrm{UfV}$ & $\mathrm{UpV}$ & $\mathrm{UqV}$ \\
\hline SFR : & 1 & 18.23 & 2.12 & 4.55 & 3.92 & 2.71 & 18.34 & 13.34 & 2.90 & 5.52 \\
TCT $[\mathrm{s}]:$ & 7.08 & 7.26 & 6.77 & 10.02 & 9.77 & 8.71 & 8.96 & 9.92 & 6.75 & 6.77 \\
\hline
\end{tabular}

The best improvements are obtained for conditions $\mathrm{V}$ (18.23-fold increase in mean SFR), TV (18.34-fold increase in SFR) and UfV (13.34-fold increase in SFR). Conditions Uf, Up and UqV yield moderate improvements (4.55-fold, 3.92-fold and 5.52-fold increase in SFR). The significantly better performance for condition V over all tactile conditions (all differences in means significant at $\mathrm{p}<0.01$ ) can be attributed to additional available information we discussed earlier as well as the higher speed of the information delivery. Pulsed vibrotactile feedback requires a certain number of pulse cycles to convey the feedback level, whereas a bargraph provides the same information instantaneously.

If this were the only factor at play, we should expect good performance from condition T. However, the observed bad performance is largely attributable to the low accuracy in condition T due to the poor clarity of the feedback. This conclusion is supported by the TCTs for the force reach and repeat tasks, which are not significantly different for conditions L, V and T (see table 2). 


\subsection{Quality of holding a target force}

\subsubsection{Mean error}

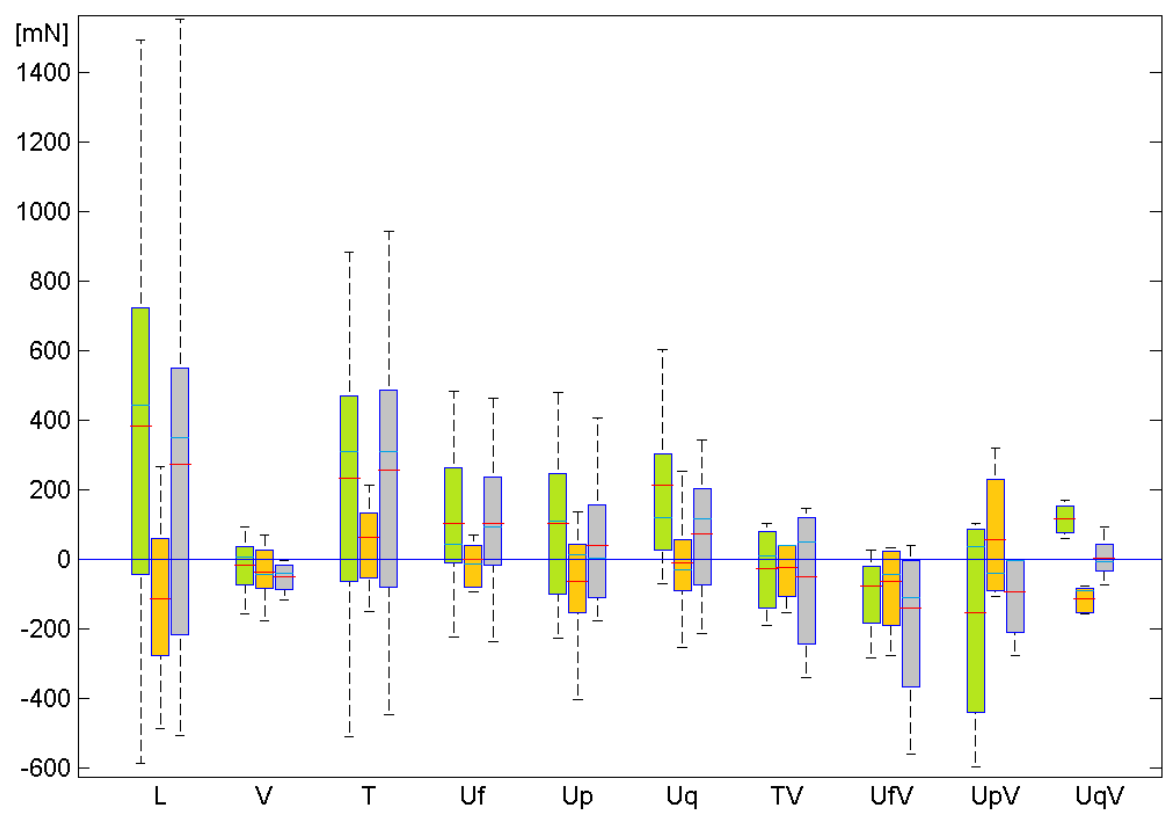

Fig. 6 Mean subject FHE (yellow boxplot), against subject DTT from the inital force reach task (green boxplot), and the resulting error (DTT+FHE), grey boxplot.

In the absence of feedback, FHE are large and subject to great spread. Condition $\mathrm{V}$ yields low FHE ( $35.3 \mathrm{mN}$ mean error) but performs on par with pulsed vibrotactile conditions (no significant differences), with Uf and Uq performing moderately better $(0.05 \mathrm{mN}$ and $10.72 \mathrm{mN}$ respectively). However, the bad performance of Uf in terms of DTT leads to an overall poor precision (when comparing mean force during force hold and the actual target force).

Table 3 Subject mean force hold error (FHE) [mN]

\begin{tabular}{lllllllllll}
\hline & $\mathrm{L}$ & $\mathrm{V}$ & $\mathrm{T}$ & $\mathrm{Uf}$ & $\mathrm{Up}$ & $\mathrm{Uq}$ & $\mathrm{TV}$ & $\mathrm{UfV}$ & $\mathrm{UpV}$ & $\mathrm{UqV}$ \\
\hline FHE : & -111.6 & -35 & 63.9 & 0.1 & -62.8 & -10.7 & -23.7 & -63.7 & 58 & -111.5 \\
FHE+DTT : & 273.2 & -49.6 & 256.2 & 102.2 & 40.3 & 75 & -48.5 & -138.2 & -94 & 4.1 \\
\hline
\end{tabular}

It would seem that tactile feedback performs comparatively well to visual feedback when it comes to avoiding drift from a given value, with an edge to pulsed 
vibrotactile feedback Uf. Continuous vibrotactile feedback is of little use in this task, with significantly worse FHE than all other tactile feedback conditions and the visual feedback condition.

Combining visual and tactile feedback yields no significant benefit over visual or tactile feedback alone. Surprisingly, condition UqV leads to significantly worse performance, which could be due to the feedback not combining in an intuitive manner. Indeed, subjects tended to report difficulties in using Uq alone, which could account for a disturbing effect when combined with visual feedback that also demands the subject's attention. Combined feedback TV yields performance on par with condition $\mathrm{V}$, leading us to believe that subjects ignored the tactile component of the combined feedback in favour of the visual feedback.

\subsubsection{Drift and cumulative deviation}
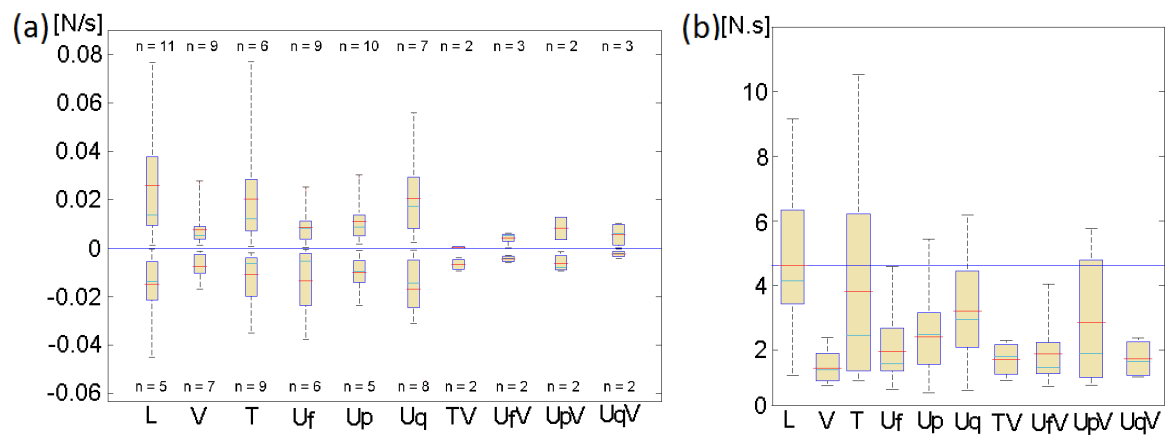

Fig. 7 (a) Subject mean drift angles $\mathrm{D} \alpha$ for force hold task [N/s]. The $\mathrm{D} \alpha \mathrm{s}$ are separated depending on the drift direction (upwards or downwards), with the number of observations for each being shown so as to give an idea of the distribution of drift directions; (b) Subject mean cumulative deviation (CD) [N.s].

As expected, the absence of feedback yields the highest $\mathrm{CD}$, due to a marked tendency to drift from $F_{\text {tgt,est }}$, with a tendency to increase the applied force over time (70\% of upward drifts against 30\% of downward drifts) and a large spread and amplitude of $\mathrm{D} \alpha$ s.

Visual feedback is more effective at reducing drift amplitude than tactile feedback alone, though not significantly. Concerning tactile feedback alone, the best performance is achieved for conditions Uf and Up (significantly outperforming $\mathrm{T}$ and $\mathrm{Uq}$ at $\mathrm{p}<0.1$ ).

All combined visual and tactile conditions significantly reduce drift and bring the mean $\mathrm{D} \alpha$ s close to $0 \mathrm{~N} / \mathrm{s}$ (equal tendency to increase and decrease force over time, lowest means and spreads of $\mathrm{D} \alpha$ values), highlighting a major benefit from combined visual and tactile feedback. In particular, condition TV shows a significant improvement over both conditions V and TV $(\mathrm{p}<0.05)$. 
Fig. 7 right shows the mean cumulative deviation from the target force for subjects during the force hold task (mean values in table 4). The lowest $\mathrm{CD}$ are obtained for condition $\mathrm{V}$ (68.8\% reduction in $\mathrm{CD}$ when compared to condition $\mathrm{L}, \mathrm{p}<0.01$, $100 \%$ of improvements over $\mathrm{L})$, closely followed by conditions Uf $(57.9 \%$ reduction in $\mathrm{CD}$ when compared to condition $\mathrm{L}, \mathrm{p}<0.01)$, TV $(62.7 \%$ reduction in $\mathrm{CD}$ when compared to condition $\mathrm{L}, \mathrm{p}<0.01)$, UfV (59.2\% reduction in $\mathrm{CD}$ when compared to condition $\mathrm{L}, \mathrm{p}<0.01)$ and $\mathrm{UqV}(62.5 \%$ reduction in $\mathrm{CD}$ when compared to condition $\mathrm{L}, \mathrm{p}<0.01$ ). None of the differences in performance between $\mathrm{V}$, Uf, TV, $\mathrm{UfV}$ and $\mathrm{UqV}$ are significant.

This shows that despite the results obtained from comparison of FHE, visual feedback and its combination with tactile feedback tends to slightly improve performance when it comes to staying close to a target over time. This can probably be attributed to the speed of delivery of the visual information when compared to the tactile information as we already mentioned in section 3.2. Once again, when observing performance for $\mathrm{TV}$, it becomes clear than the tactile component was ignored in favour of the visual feedback. Concerning UpV, the performance is worsened when compared to either of the individual forms of feedback, which may indicate a poor matching of the visual and tactile feedbacks which confused subjects.

Of the tactile feedback conditions, $\mathrm{T}$ (no significant difference to condition $\mathrm{L}$ ) and $\mathrm{Uq}$ (difference in mean significant at $\mathrm{p}<0.1$ ) perform worst, which we believe is mainly attributable to the poor understandability of these forms of feedback.

Table 4 Subject mean drift angles $(\mathrm{D} \alpha)[\mathrm{N} / \mathrm{s}]$ and cumulative deviations (CD) [N.s]

\begin{tabular}{lllllllllll}
\hline $\mathrm{L}$ & $\mathrm{V}$ & $\mathrm{T}$ & $\mathrm{Uf}$ & $\mathrm{Up}$ & $\mathrm{Uq}$ & $\mathrm{TV}$ & $\mathrm{UfV}$ & $\mathrm{UpV}$ & $\mathrm{UqV}$ & \\
\hline $\mathrm{D} \alpha$ (Up): & 0.03 & 0.008 & 0.02 & 0.009 & 0.011 & 0.021 & 0.0004 & 0.004 & 0.008 & 0.006 \\
$\mathrm{D} \alpha$ (Down): & -0.015 & -0.008 & -0.011 & -0.013 & -0.01 & -0.017 & -0.007 & -0.004 & -0.006 & -0.002 \\
$\mathrm{CD}:$ & 4.61 & 1.44 & 3.81 & 1.94 & 2.41 & 3.20 & 1.72 & 1.88 & 2.85 & 1.73 \\
\hline
\end{tabular}

\section{Conclusion}

In this paper, we describe an experiment aimed at evaluating feedback of tool-tip interaction forces in laparoscopy through haptic and/or visual cues. We compared four forms of vibrotactile feedback, on-screen visual feedback via a bar-graph and their combinations.

Providing no feedback yielded the worst overall performance for precision (strong tendency to not reach the target force, very large errors and low repeatability), speed (slowest for reaching a target force with a given accuracy) and constancy of holding a given force over time (strong tendency to increase the applied force over time, strong oscillations of force and large errors), in line with literature results on forces in LS. 
Visual feedback yielded the best performance overall. Precision and repeatability when aiming at a target force were better than those achieved through tactile feedback, and on par with or better than those achieved for combined visual and tactile feedback. Visual feedback significantly outperformed tactile feedback in terms of speed. When holding a force, it effectively allowed for reduction and correction of drift, with comparable performance to the best of tactile feedback. We believe the overall better performance of visual feedback to be due to additional information provided by the bar-graph when compared to the vibrotactile feedback as well as the comparatively higher speed of delivery of the information to the user. Vibrotactile feedback moderately improved precision. It was shown to be effective in reducing mean force errors and their spread and moderately increasing task execution speed. Concerning the quality of holding a force, the best performance in terms of drift and error reduction were achieved through pulsed vibrotactile feedback. Vibrotactile feedback involving continuous or near-continuous vibration was usually outperformed by pulsed feedback, probably due to greater user comfort.

Condition TV yielded the best results in terms of precision and speed of task execution, outperforming either $\mathrm{T}$ or $\mathrm{V}$ individually. Conditions $\mathrm{UfV}, \mathrm{UpV}$ and $\mathrm{UqV}$ yielded significant improvements over no feedback without reaching those achieved with TV, and not necessarily better than visual feedback alone, though they outperformed pulsed vibrotactile feedback alone. Concerning holding of a target force over time, combined tactile and visual feedback led to comparable errors with $\mathrm{V}$ and the best performance from tactile feedback alone, but usually significantly reduced drift when compared to $\mathrm{V}$ and tactile feedback alone respectively. It seems that pulsed vibrotactile feedback in some cases acted as a disturbance when combined with visual feedback, possibly because of discrepancies between their respective information delivery speeds.

Despite the better performance of visual feedback, these results are encouraging for the use of vibrotactile feedback in communicating force information in LS. Indeed, vibrotactile feedback clearly improves performance over no feedback and retains the advantage that it may prove effective in keeping visual cognitive load for surgeons manageable, though this remains to be investigated.

This study suffers from a few limitations, first of which being that subjects were not familiar with laparoscopy. An evaluation of the best performing forms of feedback in actual suturing trials with laparoscopic surgeons will be required. For practical reasons, the task did not take into account grasping of the thread or needle, which may impact behaviour, especially when using tactile feedback. Surgical suture knot tying is also usually a bi-manual task, so it remains to be seen whether this changes behaviour with regards to the use of feedback with implications for its design. Finally, there was no requirement to focus attention on the endoscopic image, leading to low ecological validity and likely skewing results in favour of visual feedback. Discussion with subjects showed that in conditions with visual feedback, they barely paid attention to the endoscopic image, focussing mainly on the bar-graph. We plan to address these limitations in future experiments expanding on our present work. 
Acknowledgements This work was supported by French state funds managed by the ANR within the Investissements d'Avenir programme (Labex CAMI) under reference ANR-11-LABX-0004.

\section{References}

1. Bark, K.et al.: In vivo validation of a system for haptic feedback of tool vibrations in robotic surgery. Surgical Endoscopy; 27: 65664. (2013)

2. Dankelman, J. et al.: Human Reliability and Training in Minimally Invasive Surgery. Minimally Invasive Therapy \& Allied Technologies; 12:12935. (2003)

3. Deziel, D.J. et al.: Complications of laparoscopic cholecystectomy: a national survey of 4,292 hospitals and an analysis of 77,604 cases. American Journal of Surgery; 165: 914. (1993)

4. Ding, J. et al.: Hand-assisted laparoscopic surgery versus open surgery for colorectal disease: a systematic review and meta-analysis. American Journal of Surgery; 207: 10919. (2014)

5. Joice, P. et al.: Errors enacted during endoscopic surgery - a human reliability analysis. Applied Ergonomics; 29: 40914.(1998)

6. Kitagawa, M. et al.: Analysis of Suture Manipulation Forces for Teleoperation with Force Feedback. Lecture Notes in Computer Science; 2488: 155162. (2002)

7. Kitagawa, M. et al.: Effect of sensory substitution on suture manipulation forces for surgical teleoperation. Studies in Health Technology and Informatics; 98: 15763. (2004)

8. Kitagawa, M. et al.: Effect of Sensory Substitution on Suture-Manipulation Forces for Robotic Surgical Systems. Journal of Thoracic and Cardiovascular Surgery. 129: 1518. (2005)

9. Memon, M.A. et al.: Laparoscopic Appendicectomy the current status. Annals of The Royal College of Surgeons of England; 79: 393-402 (1997)

10. Nisky, I. et al.: Perception of stiffness in laparoscopy - the fulcrum effect. Studies in Health Technology and Informatics; 173: 3139. (2012)

11. Reiley, C.E. et al.: Effects of visual force feedback on robot-assisted surgical task performance. The Journal of Thoracic and Cardiovascular Surgery; 135: 196202. (2008)

12. Schoonmaker, R.E. et al.: Vibrotactile feedback enhances force perception in minimally invasive surgery. Human Factors and Ergonomics Society 50th Annual Meeting: 10291033. (2006)

13. Sjoerdsma, W. et al:: Force transmission of laparoscopic grasping instruments. Minimally Invasive Therapy \& Allied Technologies; 6: 274278. (1997)

14. Tan, H.J. et al.: Population level assessment of hospital based outcomes following laparoscopic versus open partial nephrectomy during the adoption of minimally invasive surgery. The Journal of Urology; 191: 12317. (2014)

15. Van Der Voort, M. et al.: Bowel injury as a complication of laparoscopy. British Journal of Surgery; 91: 1253-1258. (2004)

16. Vinatier, D. et al.: What can the operator actually feel when performing a laparoscopy ? Surgical Endoscopy; 19: 95-100. (2004)

17. Weber, B. et al.: The Effects of Force Feedback on Surgical Task Performance: A Metaanalytical Integration. Haptics: Neuroscience, Devices, Modeling, \& Applications; 150-157. (2014)

18. Westebring - van der Putten, E.P. : A Sense of Touch in Laparoscopy. (Dissertation), ISBN : 9789051550719. (2011)

19. Xin, H. et al.: Laparoscopic surgery, perceptual limitations and force: A review. First CSCBC; (2006)

20. Zhou, M. et al:: Force perception threshold varies with experience in minimally invasive surgery. IEEE SMC; 2228-2232. (2007) 